\title{
DOI: https://doi.org/10.24297/jap.v17i.8538
}

\section{Site Specific Calibration Curve and Its Implication in the Treatment Planning of Head and Neck Cancer.}

\author{
${ }^{1}$ Ms. Roopam Srivastava, ${ }^{2}$ Ms. Shiva Gaur, ${ }^{3}$ Dr. P K Sharma, ${ }^{4}$ Dr Manoj Sharma, ${ }^{5}$ Dr Deepika Chauhan \\ ${ }^{6} \mathrm{Dr}$ Neha Sehgal, ${ }^{7} \mathrm{Dr}$ KJ Maria Das, ${ }^{8} \mathrm{Dr}$ Jayanand Manjhi \\ ${ }^{123456}$ Department of Radiation Oncology, IOSPL, Fortis Hospital, Noida, India \\ ${ }^{7}$ Department of Radiation Oncology, Sanjay Gandhi Post Graduate Institute, Lucknow India \\ ${ }^{8}$ Department of Biomedical Engineering, Shobhit University Merrut India \\ roopam22sep@gmail.com,roopam22sep@gmail.com
}

\begin{abstract}
Purpose: In this study, we created site specific Hounsfield Unit (HU) and Relative electron density (RED) calibration curves for computed tomography (CT) and cone beam computed tomography (CBCT) for head and neck radiotherapy dose calculation and compared them with standard calibration curves.

Methods: The calibration curves between HU and RED were generated using RANDO phantom for CT and $\mathrm{CBCT}$ and fed in the treatment planning system for dose calculation for head and neck patients. The treatment plans for 10 head and neck cancer patients were calculated using the standard calibration curves generated with CATPHAN and head and neck calibration curves generated with RANDO phantom for both CT and CBCT. Dose accuracy of head and neck calibration curves $(\mathrm{H} / \mathrm{N} \mathrm{CC})$ were compared to the standard calibration curves (SCC) using dose volume metrics and 3D gamma analysis.
\end{abstract}

Results: The results show that slightly better dosimetric agreement from the SCC can be obtained when using this $\mathrm{H} / \mathrm{N}$ CC.

Conclusions: A site specific calibration curves have been proposed and tested for the head-and-neck patients and the results explain the dosimetric benefits.

\section{Introduction}

Radiotherapy treatment planning is the process of determining the most appropriate way to treat the tumour by avoiding normal structures. One of the most important step of the planning process is the patient data acquisition required to define the target volume and normal structures and to perform accurate dose calculation [1]. These data are predominantly obtained from the patient's radiological images, primarily computed tomography (CT), which contain all the necessary information. The CT images not only provide important information about the anatomy, but also allow obtaining the distribution density of the organs and tissues. This density information is very important for accurate dose calculation, particularly in head and neck region where attenuation is very different from the attenuation in a homogeneous soft tissue volume due to tissue inhomogeneities such air and bone [2,3]. Normally, tissue density information is obtained by Hounsfield Unit(HU) and electron density calibration curve, which is generated by scanning a phantom (CATPHAN) containing various inserts of known electron density. This calibration curve is CT scanner specific and uses as standand calibration curve( $\mathrm{SCC}$ ) for dose calculation in the treatment planning system for all radiotherapy sites.[4]

For CT scans, the HU of an image set is highly dependent on many factors, including the size and material of the phantom, the materials placed in the phantom, and the imaging protocol used.[4,5]. Due to this variability, 
HU-to-density calibration curves obtained with phantoms for $\mathrm{CT}$, lack sufficient robustness to be applicable to all patients of particular site.[6]

To potentially resolve this, we developed an alternative site-specific calibration curve for head and neck patients to achieve higher dose accuracy for its possible application to adaptive radiotherapy.

A promising solution for adaptive radiotherapy is to use cone-beam computed tomography (CBCT) image sets to dosimetrically assess plan quality, since these image sets are already routinely acquired prior to treatment for patient setup and monitoring. However, to perform dose calculations, accurate tissue density information must be extracted from the CBCT voxel values. $[7,8]$

To evaluate whether this calibration curve improves the dosimetric accuracy, we performed a retrospective patient study of 10 head and- neck clinical cases, and a phantom study for both CT and CBCT.

\section{Materials and methods}

In order to validate the data, a site specific calibration curve was created using RANDO phantom (The Phantom Laboratory, NY). It is an anthropomorphic phantom made of tissue-equivalent materials. For this, Rando head phantom was scanned in the CT scanner (Gemini Philips) using same parameters as used for head and neck patient scanning. To study the HU consistency of $C B C T$ in the heterogeneous medium, $C B C T$ images were acquired using On Board Imager (OBI) system integrated with Varian Trilogy linear accelerator (Varian Medical Systems, Palo Alto, CA) with the same setup and acquisition parameters, Both the CT and CBCT image sets of the phantom were registered using rigid automatic registration. The HU values of both $\mathrm{CT}$ and $\mathrm{CBCT}$ images (same locations) at different density of head and neck area, were obtained.The phantom HU (pHU) values were compared in both CT and CBCT sets at different regions of head and neck (Table 1). The site specific head and neck $(\mathrm{H} / \mathrm{N})$ calibration curves between relative electron density (RED) and pHU values were generated for both $\mathrm{CT}$ and $\mathrm{CBCT}$ (Figure 1). Both the curves were generated separately and stored within the TPS for dose calculation.

\begin{tabular}{|l|l|l|l|}
\hline Tissue & RED & $\mathbf{p H U}_{\mathbf{C T}}$ & $\mathbf{p H U}_{\mathbf{C B C T}}$ \\
\hline Teeth & 2.67 & 2987 & 3800 \\
\hline Mandible & 1.9 & 1620 & 1820 \\
\hline Skull & 1.86 & 1480 & 1648 \\
\hline Clavicle & 1.65 & 1020 & 1085 \\
\hline Vertebrae & 1.56 & 902 & 935 \\
\hline Muscle(head) & 1.02 & 55 & 75 \\
\hline Brain & 1.02 & 40 & 65 \\
\hline Spinal cord & 1.02 & 30 & 57 \\
\hline Eye & 1.02 & 30 & 68 \\
\hline Oral mucosa, Tongue, Lips, Cheeks & 1 & 25 & 42 \\
\hline Parotids & 1 & 15 & 37 \\
\hline Nasal Passage & 1 & 2 & 10 \\
\hline
\end{tabular}




\begin{tabular}{|l|l|l|l|} 
Esophagus & 1 & 3 & 15 \\
\hline Air & 0 & -982 & -1015 \\
\hline
\end{tabular}

Table 1: Head and neck phantom HU values with their RED values for both CT and CBCT

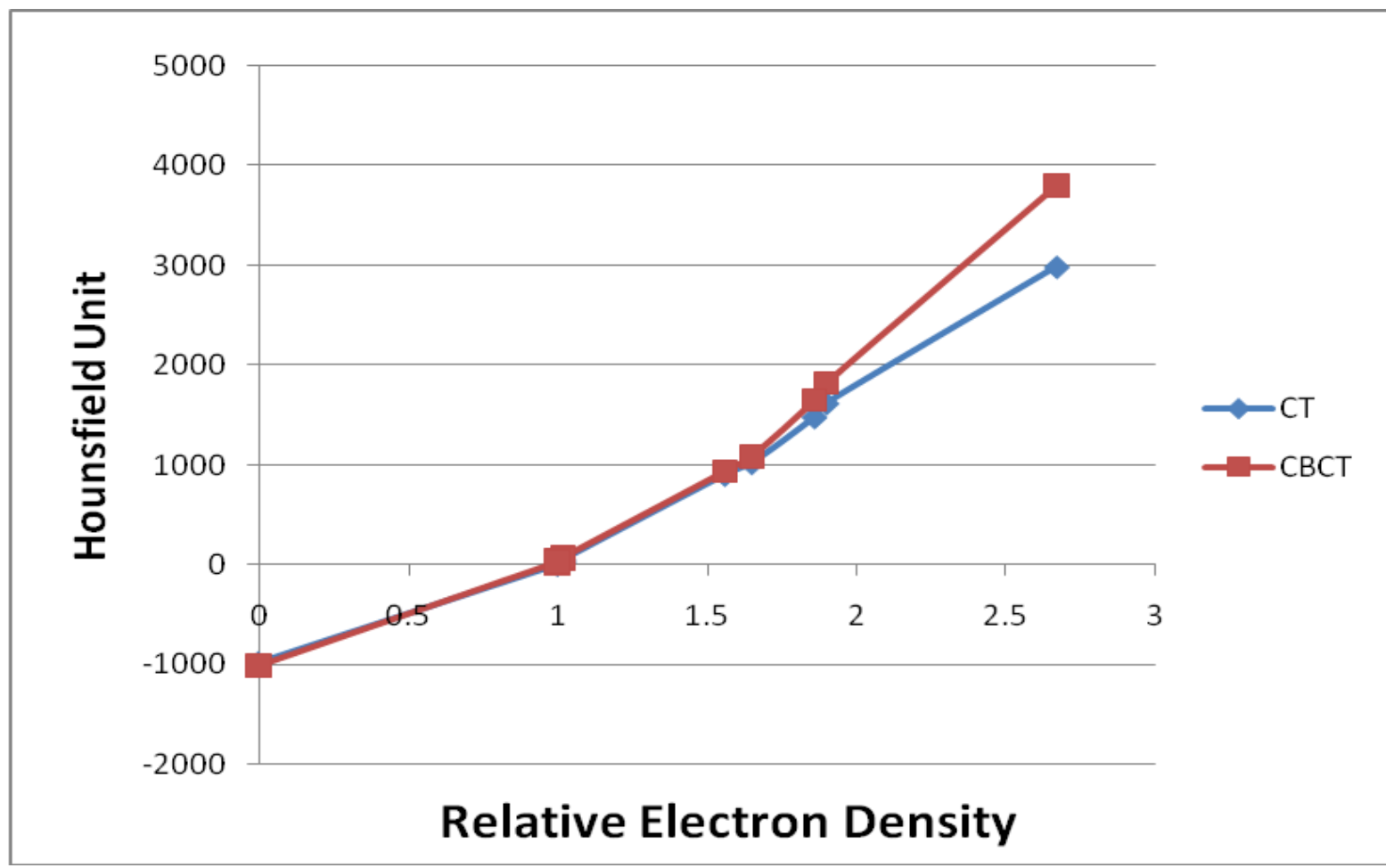

\section{Figure 1: HU and RED calibration curve for CT and CBCT using Rando head and neck phantom}

Ten head and neck cancer patients were selected at random from our institution database, all of whom had completed their treatment course .All CT and CBCT scans were acquired as a part of our routine clinical work. A nine-field $6 \mathrm{MV}$ photon IMRT plan was optimized on CT scan of each patient for 70Gy in 35 fractions and calculated using Analytical Anisotropic Algorithm(AAA) with both standard calibration curve(SCC) and head and neck calibration curve (H/N CC). To run the optimization, structure set : clinical target volume (CTV) expanded with margin to a planning target volume (PTV), spinal cord, parotid glands, and mandible were delineated. $C T$ and $C B C T$ image sets were rigidly registered and the structure set was copied from $C T$ to $C B C T$ with a small manual adjustments in targets volumes (CTV,PTV) and Organs at Risk (OARs). Subsequently, the IMRT plan with fluence maps and monitor units was copied from $C T$ to the $C B C T$ and calculated using $C B C T$ SCC and CBCT H/N CC.

The treatment plans with $\mathrm{SCC}$ and $\mathrm{H} / \mathrm{N} \mathrm{CC}$ for both $\mathrm{CT}$ and $\mathrm{CBCT}$ were evaluated by comparing dose-volume histogram (DVH) of PTV and OARs (Spine, mandible, parotids). To evaluate the dose distributions the minimum, maximum and mean dose from DVH parameters of the structures were recorded. Finally, the complete three-dimensional dose distributions were compared using gamma-index pass/fail analysis using $3 \%$ dose difference and $3 \mathrm{~mm}$ distance-to-agreement criteria.

\section{Results}

The results exhibit improved target coverage using $\mathrm{H} / \mathrm{N} \mathrm{CC}$ for both $\mathrm{CT}$ and $\mathrm{CBCT}$. Doses for spine, and parotids were significantly less whereas mandible dose was significantly higher in plans using $\mathrm{H} / \mathrm{N} \mathrm{CC}$ as compared to SCC(Table 2). The results of the 3D gamma analysis were found to be similar for both the curves 
( $P$ value $=0.15)$, with the average (standard deviation) gamma pass rates of $96.7 \%(3.0 \%)$ and $96.1 \%(3.3 \%)$ for the $\mathrm{H} / \mathrm{N} \mathrm{CC}$ and SCC respectively.

\begin{tabular}{|ll|l|l|l|l|l|l|l|}
\hline \multirow{2}{*}{ Treatment plans } & \multicolumn{2}{|l|}{ PTV doses (Gy) } & \multicolumn{3}{l|}{ OAR doses (Gy) } \\
\cline { 2 - 9 } & & Dmax & Dmean & Dmin & $\begin{array}{l}\text { Spine } \\
\text { Dmax }\end{array}$ & $\begin{array}{l}\text { Mandible } \\
\text { Dmax }\end{array}$ & $\begin{array}{l}\text { Rt Parotid } \\
\text { Dmean }\end{array}$ & $\begin{array}{l}\text { Lt Parotid } \\
\text { Dmean }\end{array}$ \\
\hline $\begin{array}{l}\text { CT } \\
\text { using SCC }\end{array}$ & Plan & $74.3 \pm 2.3$ & $\begin{array}{l}70.9 \pm 1 . \\
4\end{array}$ & $66.8 \pm 1.1$ & $42.3 \pm 1.9$ & $71.4 \pm 0.8$ & $23.9 \pm 16.2$ & $32.3 \pm 20.5$ \\
\hline $\begin{array}{l}\text { CT } \\
\text { using H/N CC }\end{array}$ & Plan & $76.2 \pm 3.5$ & $\begin{array}{l}71.3 \pm 0 . \\
8\end{array}$ & $68.1 \pm 0.9$ & $42.0 \pm 0.8$ & $72.5 \pm 1.3$ & $22.7 \pm 19.3$ & $31.0 \pm 18.1$ \\
\hline $\begin{array}{l}\text { CBCT } \\
\text { using SCC }\end{array}$ & Plan & $75.4 \pm 2.8$ & $\begin{array}{l}71.1 \pm 1 . \\
6\end{array}$ & $67.0 \pm 1.1$ & $44.4 \pm 1.7$ & $72.8 \pm 1.2$ & $28.9 \pm 18.2$ & $30.7 \pm 18.7$ \\
\hline $\begin{array}{l}\text { CBCT } \\
\text { using H/N CC }\end{array}$ & Plan & $76.7 \pm 1.9$ & $\begin{array}{l}71.5 \pm 1 . \\
2\end{array}$ & $67.7 \pm 2.1$ & $43.3 \pm 2.1$ & $74.5 \pm 0.3$ & $27.4 \pm 16.6$ & $28.8 \pm 17.6$ \\
\hline
\end{tabular}

Table 2: Dosimetric data from treatment plans calculated on $\mathrm{CT}$ and $\mathrm{CBCT}$ using SCC and H/N CC

\section{Discussion}

The calibration for HU to RED conversion in a treatment planning system (TPS) is typically based on measurements in CT scans of tissue equivalent materials of known electron densities in a phantom. Yang et al. investigated the stability of this tissue substitute HU-RED calibration for CBCT-based dose calculation. Based on CBCT scans of the Catphan 504 phantom (The Phantom Laboratory, Salem, NY, USA), supplied by the vendor for linear attenuation to HU calibration and image quality assessment, the HU-RED curve has been found to be stable both over time and across different scan settings[10] . However, the phantom uses Teflon composed of carbon and fluorine as the high electron density substitute for dense bone. In real bone the presence of calcium and phosphor with higher effective atomic number results in higher $\mathrm{HU}$ values for the same electron density as Teflon due to more photoelectric interactions leading to dose calculation inaccuracies[11,12]. More importantly for the variation in HU-RED relation for CBCT is the amount of scatter present during calibration which should resemble the clinical treatment situation. Hatton et al. shown a large impact on $\mathrm{HU}$ numbers due to the phantom length and especially the radial diameter for the head and neck region[8]. Also a reduction in scan length or cone angle will reduce the scatter contribution and change the HU-RED curve[13]. To minimize the influence on dose calculation accuracy site-specific HU-RED calibrations has been implemented. This has aimed to avoid the use of tissue equivalent materials by creating patient specific HU-RED curves. The average CBCT HU values for clinical tissue types were determined and subsequently mapped to average $\mathrm{CT}$ electron density for the same tissues.

Unlike the CT-based planning that uses only a single CT to electron density calibration curve(SCC) in the treatment planning system, a site-specific HU-to-density curve was created for each head and neck patient and compared it with SCC. HU to RED standard calibration curves for CT and CBCT were generated using CATPHAN and are using in our centre for all routine cases for treatment planning. James et al showed a variation of $\pm 14 \%$ of the $\mathrm{pCT}$-based dose using CATPhan phantom and the CT calibration curve[14]. The RANDO phantom is reliable for HU to RED for performing daily plans because it is made with three different 
materials (skeleton of normal human,soft tissue,lung) in an effort to overcome the disadvantages of nonuniformity of materials, size and shape. Dosimetric calculations on CT/CBCT images show doses with variations within $\pm 5 \%$ of the CT-based plans, using the RANDO phantom. This concludes that while the CATPhan phantom produces linear responses and produces highly accurate and consistent $\mathrm{HU}$ values on $\mathrm{CT} / \mathrm{CBCT}$, it does not provide accurate dosimetric values as compared to RANDO Phantom.

We found that $\mathrm{CT}$ number deviations are minimal for low-density materials but become significant for highdensity materials, therefore, high-density materials may have a large effect on the accuracy of CT number and dose calculation. Recently Zurl et al [15] compared CT parameters and showed HU variation upto $20 \%$ with the impact on dose to $1.5 \%$.

The results show that slightly better dosimetric agreement with the SCC can be obtained when using this $\mathrm{H} / \mathrm{N}$ CC, for plan re-assessment during radiotherapy.

\section{Conclusion}

A site specific $C T$ and $C B C T$ calibration curves have been proposed and tested for the head-and-neck site.It is recommended to implement site specific curves for sites other than head and neck especially in lung cases to look upon the dosimetric accuracy.

\section{Disclosures}

No conflicts of interest, financial or otherwise, are declared by the authors.

\section{Ethical Statement}

This is a prospective study, therefore, no testing on humans and animals .All the patients were treated as per protocol for which written informed consent has been obtained from all patients. This dosimetric study was done on the images obtained during treatment.

\section{References}

1. W. Parker, H. Patrocinio. "Clinical Treatment Planning in External Photon Beam Radiotherapy." In: E. B. Podgorsak (Ed.). Radiation Oncology Physics: A Handbook for Teachers and Students. Austria: IAEA, p. 229-273, 2005.

2. A. Neal. "Patient Data Acquisition." In: P. Mayles, A. Nahum, J. C. Rosenwald (Org.). Handbook of Radiotherapy Physics: Theory and Practice. Inglaterra: Taylor \& Francis, p.647-657, 2007.

3. A. Barret, J. Dobbs, S. Morris, T. Roques. Practical Radiotherapy Planning. 2009.

4. Saw CB, Loper A, Komanduri K, Combine T, Huq S, Scicutella C. Determination of CT-to-density conversion relationship for image based treatment planning systems. Med Dosim. 2005;30:145-148.

5. Cozzi, L.; Fogliata, A.; Buffa, F.; et al. Dosimetric impact of computed tomography calibration on a commercial treatment planning system for external radiation therapy. Radiother. Oncol. 48: 335-8; 1998.

6. Constantinou, C.; Harrington, J.; Dewerd, L. An electron-density calibration phantom for CT-based treatment planning computers. Med. Phys. 19:325-7; 1992.

7. Yoo S, Yin FF. Dosimetric feasibility of cone-beam CT-based treatment planning compared to CT-based treatment planning. Int J Radiat Oncol Biol Phys. 2006;66:1553-1561. 
8. Hatton J, McCurdy B, Greer PB. Cone beam computerized tomography: the effect of calibration of the Hounsfield unit number to electron density on dose calculation accuracy for adaptive radiation therapy. Phys Med Biol. 2009;54:N329-N346.

9. Richter $A, \mathrm{Hu} Q$, Steglich $\mathrm{D}$, et al. Investigation of the usability of cone beam $C T$ data sets for dose calculation. Rad Oncol. 2008;3:42.

10. Yang Y, Schreibmann E, Li T, Wang C, Xing L. Evaluation of on-board kV cone beam CT (CBCT)-based dose calculation.Phys Med Biol 2007;52:685-705.

11. Verhaegen F, Devic S. Sensitivity study for CT image use in Monte Carlo treatment planning. Phys Med Biol 2005;50: 937-46.

12. Vanderstraeten B, Chin PW, Fix M, Leal A, Mora G, Reynaert N, et al. Conversion of CT numbers into tissue parameters for Monte Carlo dose calculations: A multicentre study. Phys Med Biol 2007;52:539-62.

13. Guan $\mathrm{H}$, Dong $\mathrm{H}$. Dose calculation accuracy using conebeam $\mathrm{CT}$ (CBCT) for pelvic adaptive radiotherapy. Phys Med Biol 2009;54:6239-50.

14. James Kwame Annkah, Ivan Rosenberg et al.Assessment of the dosimetric accuracies of CATPhan 504 and CIRS 062 using kV-CBCT for performing direct calculations. J Med Phys. 2014 Jul-Sep; 39(3): 133-141.

15. Zurl $B$, Tiefling $R$, Winkler $P$, Kindl $P$, Kapp KS. Hounsfield units variations: Impact on $C T$-density based conversion tables and their effects on dose distribution. Strahlenther Onkol. 2014;190:88-93. 